\title{
A MODEL FOR PRODUCING STABLE, BROADBAND TERAHERTZ COHERENT SYNCHROTRON RADIATION IN STORAGE RINGS*
}

\author{
F. Sannibale ${ }^{1}$, J. M. Byrd ${ }^{1,2}$, A. Loftsdottir ${ }^{1,2}$, M. C. Martin ${ }^{1}$, M. Venturini ${ }^{1}$, \\ ${ }^{1}$ Lawrence Berkeley National Laboratory, One Cyclotron Road, Berkeley, California 94720 \\ ${ }^{2}$ Department of Physics, University of California, Davis, Davis, California 95616
}

\section{Abstract}

We present a model for producing stable broadband coherent synchrotron radiation (CSR) in the terahertz frequency region in an electron storage ring. The model includes distortion of bunch shape from the synchrotron radiation (SR), enhancing higher frequency coherent emission and limits to stable emission due to a microbunching instability excited by the SR. We use this model to optimize the performance of a source for CSR emission.

\section{INTRODUCTION}

Coherent synchrotron radiation (CSR) occurs when the synchrotron emission from a bunch of relativistic electrons is in phase. In this regime the radiation intensity is proportional to the square of the number of particles per bunch in contrast to the linear dependence of the conventional incoherent radiation. Considering that the number of particles per bunch is typically very large $\left(>10^{6}\right)$, the potential intensity gain for a CSR source is huge. Although CSR was predicted to occur in high energy storage rings over 50 years ago [1], it is only recently that steady state CSR has been observed for the first time in the BESSY-II storage ring [2, 3]. Coupled with the first successful application of such a source [4], there is the exciting possibility of using this radiation as an innovative and very powerful source in the far infrared/terahertz frequency range.

Attractive features of the BESSY-II radiation were a flux increase of about five orders of magnitude over the incoherent synchrotron spectrum, a broadband frequency range from about $3 \mathrm{~cm}^{-1}$ to $60 \mathrm{~cm}^{-1}$ and the stability of the source. However, several curious features of the radiation were also observed. First, the coherent emission spectrum extended to shorter wavelengths than expected from a Gaussian electron bunch of the measured length. Second, a significantly non-Gaussian longitudinal distribution of the electron bunch was observed. Third, a threshold current was observed above which the CSR was emitted in chaotic bursts. We have developed a model that accounts for the above-mentioned observations and provides a tool for predicting and optimizing the performance of a ring-based CSR source.

*Work Supported by the Director, Office of Science, of the U.S. Department of Energy under Contract No. DE-AC03-76SF00098
This paper presents the elements of the model and describes the design criteria for an optimized stable ring based CSR source. We consider only the case of a bending magnet.

\section{CSR RADIATION AND SR WAKEFIELD}

The synchrotron radiation (SR) power spectrum is given by $[1,5]$ :

$$
\frac{d P}{d \lambda}=\frac{d p}{d \lambda}[N+N(N-1) g(\lambda)]
$$

where $\lambda$ is the wavelength of the radiation, $p$ is the single particle emitted power, $N$ is the number of particles per bunch and $g$ is the so-called CSR form factor. This numerical quantity, whose value ranges between 0 and 1 , is essentially the square of the Fourier transform of the normalized bunch distribution. In expression (1) both the incoherent and coherent terms are included. The first is simply the part proportional to $N$ while the second is proportional to $N^{2}$ and to $g$. In order to have significant CSR emission at the wavelength of interest it must be $g(\lambda)>1 / N$. For short wavelengths, according to the definition of $g$, this implies having short bunches. For longer wavelengths $g$ increases and approaches 1 but above the vacuum chamber cutoff wavelength the CSR emission is quickly inhibited. For the particular case of a Gaussian distribution, CSR occurs for $\sigma_{z}<\lambda \pi$, where $\sigma_{z}$ is the rms bunch length. In the general case, comparing at short wavelengths bunches with same rms length, non Gaussian distributions can present a $g$ factor significantly larger than Gaussian ones. This implies that these 'distorted' bunches can have CSR emission at wavelengths remarkably shorter than in the purely Gaussian case. From the experimental results on references [2] and [3] it can be found that the BESSY-II CSR emission belongs to this category. In fact, streak camera measurements indicated bunch lengths $\sim 1 \mathrm{~mm}$ while CSR was visible down to $\lambda \sim 200 \mu \mathrm{m}$, in disagreement with the condition for Gaussian bunches and indicating the presence of distorted distributions.

Equilibrium longitudinal distributions in electron storage rings are usually Gaussian. The possible phenomena that can generate bunch distortions can be classified in two main categories. Non linear dynamics 
effects and wakefield induced effects. RF and lattice nonlinearities belong to the first group, while CSR and vacuum chamber wakefields fall in the second one. For most of the storage rings, including BESSY-II, RF nonlinearities are very small and can be neglected. We have simulated the effects of lattice nonlinearities for the case of BESSY-II. The results showed a very small distortion effect that was clearly insufficient to explain the experimental data. In the wakefield category, the SR wakefield, which is always present, is the natural candidate for beginning this analysis and a reasonable approach consists in starting with the simplest model, the free space electron SR wakefield $[6,7,8]$ :

$$
W(\tau)=\frac{e}{2 \pi \rho} \frac{d S(\tau)}{d \tau}=\frac{e}{2 \pi \rho} \frac{d}{d \tau}\left(-Z_{0}\left(\frac{\rho}{3 c}\right)^{1 / 3} \tau^{-1 / 3}\right)
$$

where $Z_{0}=377$ ohms, $\rho$ is the dipole magnet bending radius, $c$ is the speed of light, $e$ is the electron charge and $\tau$ is the distance from the wake inducing particle in time units. For $\tau<0 \quad W(\tau)=0$, indicating that in the SR wakefield case particles in the head of the bunch cannot influence the ones in the tail. The free space assumption can be justified by the fact that for bunches much shorter than the vacuum chamber height, and an optimized CSR source must be designed to fulfill this requirement, the shielding effects are small.

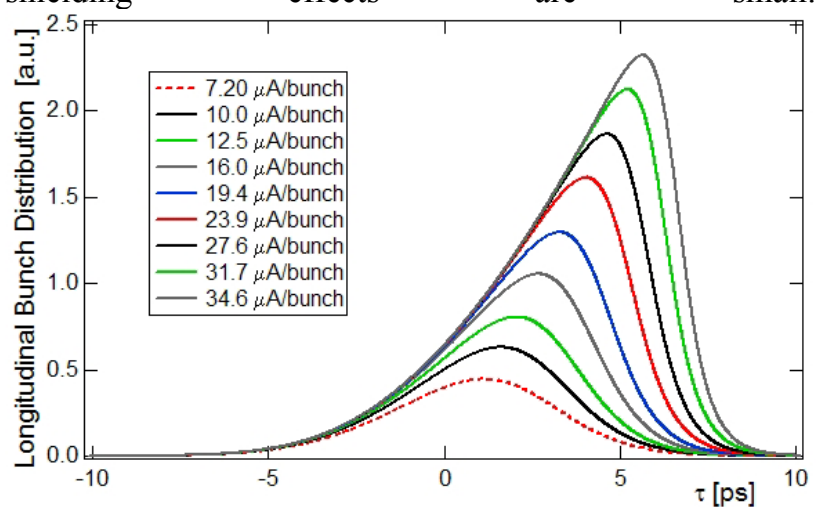

FIG. 1. Calculated equilibrium longitudinal distribution for different currents per bunch using the free space SR wakefield. BESSY-II case.

This free space SR case was already investigated in reference [8] where the Haissinski equation solution for the longitudinal current distribution $I(\tau)$ was numerically calculated:

$$
I(\tau)=K e^{-\frac{(c \tau)^{2}}{2 \sigma_{z}^{2}}-\frac{c^{2}}{\sigma_{z}^{2} \dot{V}_{R F}} \int_{0}^{\infty} I(\tau-t) S(t) d t}
$$

where $\sigma_{z}$ is the natural bunch length, $\dot{V}_{R F}$ is the time derivative of the radio frequency (RF) voltage at the synchronous phase and $K$ is a normalization constant.

Figure 1 shows, in the example of BESSY-II, the equilibrium longitudinal bunch profile calculated by this model for different currents per bunch. A strong distribution asymmetry (sharper leading edge) increasing with the bunch current and thus with the SR wake is clearly visible. The result demonstrates that the SR wakefield alone is able to produce non-Gaussian bunches that significantly enhance the CSR emission towards shorter wavelengths as shown in Figure 2.

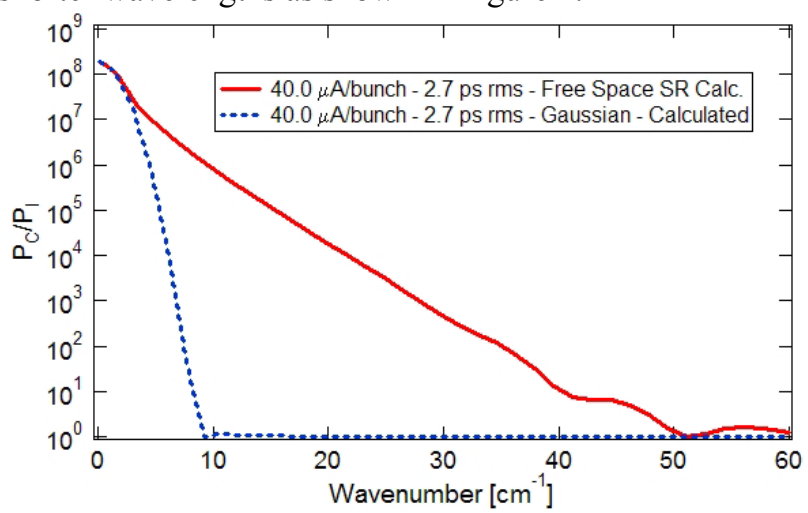

FIG. 2. Comparison between the CSR gain (ratio between coherent and incoherent SR power) for the two cases of Gaussian and SR wakefield distorted distribution for same current and bunch length. BESSY II parameters.

\section{OPTIMIZING A STORAGE RING BASED CSR SOURCE}

We want now to design a source exploiting this effect. In the design of such a source one of the fundamental requirements is the stability of the CSR emission. A SR driven single bunch instability has been theoretically predicted [9], simulated [10] and experimentally verified [11]. Above a current threshold the beam becomes unstable and transient longitudinal microstructures appears generating CSR bursts. In the following source optimization analysis, stability criteria are included. In the free space SR model, the number of particles per bunch at equilibrium is given by [8]:

$$
N=A \frac{B^{1 / 3} f_{R F} V_{R F} \sigma_{z}^{7 / 3}}{E^{1 / 3}} F
$$

where $A=6.06810^{-4}$ [MKS units], $B$ is the dipole magnet magnetic field, $f_{R F}$ is the storage ring RF frequency, $V_{R F}$ the RF peak voltage, $E$ is the beam energy, $\sigma_{z}$ is the natural bunch length and $F$ is a dimensionless quantity proportional to the integral of the bunch distribution. $F$ and the bunch distribution are calculated by the free space SR model. The larger the current per bunch the stronger is 
the distribution distortion and the larger is $F$. Using equation (1) for $N g \gg>1$ and the expression for $d p / d \lambda$ when the wavelength is much larger than the critical wavelength (see for example [12]) we can write for a ring with $N_{b}$ bunches:

$$
\frac{d P}{d \lambda}=C N_{b} \frac{B^{1 / 3} f_{R F}^{2} V_{R F}^{2}}{L E^{1 / 3}} \frac{\sigma_{z}^{14 / 3}}{\lambda^{7 / 3}} F^{2} g(\lambda)
$$

where $C=2.64210^{-21}$ [MKS units] and $L$ is the storage ring length. In optimizing a CSR source we want to pursue the following tasks: increase as much as possible the band of wavelengths where the SR emission is coherent, maximize expression (5) and maintain the beam stability. The bandwidth of the CSR emission can be extended towards longer wavelengths by designing high cutoff frequency vacuum chambers. On the other side, as we said, the bandwidth can be extended towards shorter wavelengths by shortening the bunch and/or by increasing the bunch distortion. The second way is strongly preferred because it increases $F$ and according to Eqn. (5) also the CSR power. On the other hand, Eqn. (5) shows that decreasing the bunch length dramatically decreases the power. The distortion is enhanced by increasing the number of particles per bunch but the maximum value that this quantity can assume is limited by the previously mentioned microbunching instability $[9,11]$ :

$$
N \leq N_{S}=D \frac{B^{1 / 3} f_{R F} V_{R F}}{E^{1 / 3}} \frac{\sigma_{z}^{3}}{\lambda^{2 / 3}}
$$

where $D=4.528 \quad 10^{-3}$ [MKS units]. By combining equations (4) and (6) the following stability criterion can be derived:

$$
F \leq F_{M A X}=\frac{1}{G}\left(\frac{\sigma_{z}}{\lambda}\right)^{2 / 3}
$$

where $G=0.1340$ is a dimensionless constant. It must be remarked that the microbunching instability theory was derived for the case of a coasting beam. Anyway simulations and experimental results at the ALS [11] showed that the model works also for bunched beams and that the theory is able to predict the instability threshold when in Eqn. (7) $\lambda \sim 2 \sigma_{z}$ is used obtaining $F_{M A X} \sim 4$.7. In the case that $\sigma_{z}$ is greater than the vacuum chamber cutoff wavelength $\lambda_{C O}$, then $\lambda=\lambda_{C O}$ must be used in Eqn. (7). The maximum number of stable particles per bunch can be evaluated by using $F_{M A X}$ in Eqn. (4). Once the bandwidth optimization is completed the quantities $\sigma_{z}$, $F$ and $g(\lambda)$ in Eqn. (5) are frozen and cannot be modified anymore. All the remaining 'knobs' in Eqn. (5) can be freely changed, within the technological limits, for the maximization of the CSR power. It must be remarked that the momentum compaction, which does not appear explicitly in Eqn. (5), is used in this scheme for keeping a constant $\sigma_{z}$ when the other quantities are changing. Figure 3 shows an example of the impressive performances that a source designed with the presented criteria can achieve.

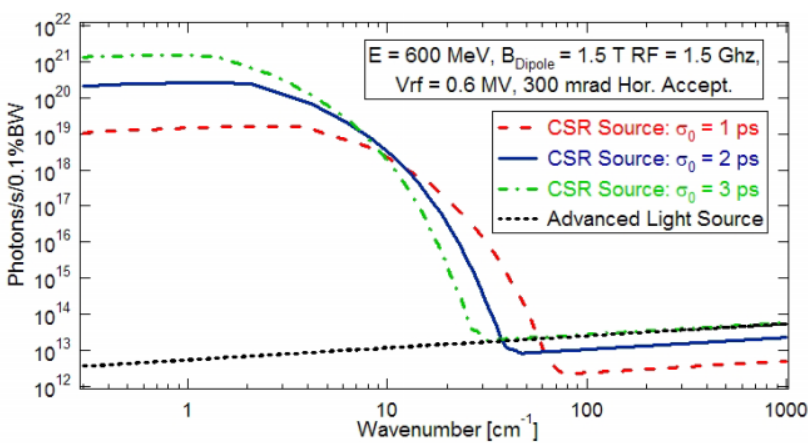

FIG. 3. Example of source optimized for the CSR production using the criteria described in this letter. The potential photon flux gain in the Far-IR THz region of the spectrum is huge if compared with an existing conventional source (ALS).

Such a source would have between 6 and 8 orders of magnitude greater average flux than other available sources in the $\mathrm{THz}$ and sub- $\mathrm{THz}$ frequency range.

\section{ACKNOWLEDGEMENTS}

The authors would like to thank D. Robin for his continuous support and fruitful discussions, E. Forest for his collaboration in investigating the lattice nonlinearities effects and J. Murphy, B. Warnock, P. Kuske and G. Wustefeld for useful discussions.

\section{REFERENCES}

[1] J. S. Nodvick and D.S. Saxon, Phys. Rev. 96, 180 (1954)

[2] M. Abo-Bakr et al., Phys. Rev. Lett. 88, 254801 (2002).

[3] M. Abo-Bakr et al., Phys. Rev. Lett. 90, 094801 (2003).

[4] J. Singley et al., submitted to Phys. Rev. Lett.

[5] G. Williams et al., Phys. Rev. Lett. 2, 261 (1989).

[6] J. B. Murphy, S. Krinsky, and R. L. Gluckstern, Part. Accel. 57, 9 (1997).

[7] Y. S. Derbenev, et al., DESY Report No. TESLA-FEL 9505, 1995.

[8] K. Bane, S. Krinsky, and J. B. Murphy, in Micro Bunches Workshop, Upton, NY, 1995, edited by E. B. Blum, M. Deines, and J. B. Murphy, AIP Conf. Proc. 367 (AIP, Woodbury, NY, 1996), p. 191.

[9] S. Heifets and G. Stupakov, Phys. Rev. ST Accel. Beams 5, 054402 (2002).

[10] M. Venturini and R. Warnock, Phys. Rev. Lett. 89, 224802 (2002).

[11] J. M. Byrd, et al., Phys. Rev. Lett. 89, 224801 (2002).

[12] A. Hofmann, Synchrotron Radiation and Free Electron Lasers CAS, CERN 98-04. 